\title{
Brain Tumor Detection using a Combination of Bayesian Optimization Based SVM Classifier and Fine-Tuned Based Deep Features
}

\author{
Muammer Türkoğlu ${ }^{1 *}$ \\ 1* Samsun University, Faculty of Engineering, Departmant of Software Engineering, Samsun, Turkey, (ORCID: 0000-0002-2377-4979), \\ muammer.turkoglu@samsun.edu.tr
}

(First received 7 July 2021 and in final form 9 September 2021)

(DOI: 10.31590/ejosat.963609)

ATIF/REFERENCE: Turkoglu, M. (2021). Brain Tumor Detection using a Combination of Bayesian Optimization Based SVM Classifier and Fine-Tuned Based Deep Features. European Journal of Science and Technology, (27), 251-258.

\begin{abstract}
Brain tumor, one of the most common types of cancer, is a fatal disease. Therefore, accurate diagnosis of this disease and determining the type of tumor are of great importance in terms of early treatment. In this context, research, and interest in the development of automatic systems for the problems experienced in brain tumor classification, based on deep learning, have increased recently. In this study, a unique framework is proposed, which is based on Bayesian optimization-based Support Vector Machine (SVM) classifier and Convolutional Neural Network (CNN) based deep features ensemble, for the classification of brain tumors. In this model, brain MRI images are first improved. Second, the deep features are extracted using pre-trained CNN-based deep architectures and then combined. Later, effective, and distinctive features are selected from these deep features with the MrMr algorithm. Finally, these features are used in the training of the SVM classifier based on the Bayesian optimization algorithm. A dataset named Figshare, containing brain tumor images such as meningioma, glioma, and pituitary, is used to test the proposed system. In the experimental studies, the accuracy score of the model proposed was observed to be more successful than that of the other studies.
\end{abstract}

Keywords: Brain Tumor Classification, Feature selection, Convolutional Neural Network, Support Vector Machine, Bayesian optimization

\section{Bayes Optimizasyon Tabanlı SVM Sınıflandırıcı ve İnce-Ayar Tabanlı Derin Özelliklerinin Kombinasyonu Kullanılarak Beyin Tümörü Tespiti}

$\ddot{O} \mathbf{z}$

En sık görülen kanser türlerinden biri olan beyin tümörü ölümcül bir hastalıktır. Bu nedenle bu hastalığın doğru teşhisi ve tümörün tipinin belirlenmesi erken tedavi açısından büyük önem taşımaktadır. Bu bağlamda, son zamanlarda beyin tümörü sınıflandırmasında yaşanan problemler için derin öğrenmeye dayalı otomatik sistemlerin geliştirilmesine yönelik araştırmalar ve ilgi artmıştır. Bu çalışmada, beyin tümörlerinin sınıflandırılması için Bayesian optimizasyon tabanlı Destek Vektör Makinesi (DVM) sınıflandırıcısı ve Evrişimsel Sinir Ağı (ESA) tabanlı derin öznitelikler topluluğuna dayalı benzersiz bir tasarım önerilmiştir. Bu modelde öncelikle beyin MRI görüntüleri iyileştirildi. İkinci olarak, derin öznitelikler, önceden eğitilmiş ESA tabanlı derin mimariler kullanılarak çıkartıldı ve ardından birleştirildi. Daha sonra, MrMr algoritması ile bu derin özelliklerden etkili ve ayırt edici özellikler seçildi. Son olarak, bu özellikler, Bayes optimizasyon algoritmasına dayalı DVM sınıflandırıcısının eğitiminde kullanıldı. Önerilen sistemi test etmek için, meningiom, glioma ve hipofiz gibi beyin tümörü görüntülerini içeren Figshare adlı bir veri seti kullanıldı. Deneysel çalışmalarda, önerilen modelin doğruluk skoru diğer çalışmalardan daha başarılı olduğu gözlemlenmiştir.

Anahtar Kelimeler: Beyin Tümör Sınıflandırımlası, Özellik Seçimi, Evrişimsel Sinir Ağları, Destek Vektör Makinesi, Bayes Optimizasyon.

\footnotetext{
*Corresponding Author: muammer.turkoglu@ samsun.edu.tr
} 


\section{Introduction}

Worldwide, the number of deaths from brain tumors is increasing day by day (Ari, 2019). A brain tumor can be categorized into one of two types, benign or malignant. Early diagnosis of brain tumors and determination of the tumor type are critical for increasing survival rates and effective treatment options (Afshar et al., 2019).

Magnetic Resonance Imaging (MRI) is an important medical imaging tool for the detection of brain tumor (Amin et al., 2019; Amin et al., 2020). Determination of the type and detection of tumors using MRI devices is performed by experts. This identification process is a time-consuming process and entails a high margin of error. The reason for this is that tumors have different shapes and sizes, and they can also be found in different parts of the brain. In addition, manual detection of brain tumor is not a practical method in cases where the number of patients is high. For all these reasons, there is a need for automatic detection of brain tumors. Recently, systems developed to meet this need based on computer vision and machine learning are on demand. Thanks to computer-aided systems that help experts, accurate and rapid diagnosis of brain tumors has been achieved (Ari, 2019).

In recent years, many studies have been performed for classification of the brain tumor based on MRI images. In these studies, CNN-based deep learning models, and machine learning methods were widely used. In this regard, Ali et al. (2020) proposed a model based on pre-trained deep architectures and ELM classifier for brain tumor classification. In this study, deep features are extracted using the fc6 and fc7 layers of AlexNet and VGG16 models. Later, these feature vectors were used for classification of MR images with the help of Extreme Learning Machines (ELM) classifier. They reported a highest accuracy score of $97.64 \%$. Similarly, Rehman et al. (2020) developed a deep learning-based framework using transfer learning method for classification of brain tumors. In line with this purpose, they used three architectures of convolutional neural networks (AlexNet, GoogLeNet, and VGGNet). In addition, the dataset was expanded using data augmentation methods. They achieved a highest accuracy score of $98.69 \%$ using the fine-tune VGG16 architecture. Cheng et al. (2016) proposed a unconventional feature extraction framework to enhance the classification performance. They extracted features using Adaptive Spatial division and Fisher Vector Representation. Later, SVM classifier was used for training the model proposed and the performance of the system was calculated. In this study, mean precision value was obtained as $94.68 \%$. Abir et al. (2018) developed a system based on pre-processing methods and GLCM (Gray Level Cooccurrence Matrix) to extract the feature values. The features obtained were used as the input of a PNN classifier and the performance of the system proposed was calculated. In this study, the highest accuracy acore was obtained as $83.33 \%$. Finally, Afshar et al. (2018) used Capsule networks (CapsNets) for the problem encountered in classification of brain tumor type. They made investigations to increase the accuracy by changing the number of feature maps in the convolutional layer of the Capsule network. The results of this study show that the method proposed achieved an accuracy score of $86.56 \%$. Additionally, other previous studies used the Figshare dataset are summarized in the Discussion section.

In this paper, a unique Bayesian optimization based deep classifier approach is proposed for classification of brain tumors. Firstly, image enhancement techniques were used in the system proposed. Later, deep features were extracted from brain tumor images using pre-trained deep architectures based on the transfer learning approach. Effective and distinctive features were selected using MrMr method for these representative features. Finally, parameters that had the best performance of the SVM classifier based on the Bayesian optimization method were determined and the training and testing phases of the system proposed were carried out. A brain tumor dataset named Figshare (Cheng, 2018) was used to evaluate the performance of the system proposed.

The main contributions of this study are as follows:

- The proposed model used classification capabilities of features obtained from the last fully connected layers of pretrained deep architectures: AlexNet, and DenseNet201.

- The current study adapted the MrMr feature selection algorithm in order to both reduce the dimension of the features obtained from pre-trained deep networks and reveal the best effective deep features. In experimental studies, a high success diagnosis model has been achieved by using these selected and effective features for brain tumor MRI classification.

- The deep features obtained from pre-trained CNN networks were fed into an SVM, and the best SVM classifier parameters have been optimized using the Bayesian optimization method. Thanks to this approach, the highest performance has been achieved as $98.04 \%$ for the SVM classifier based on selected features in classifying brain tumors.

The remaining part of this paper is structured as follows: the proposed approach is presented in Section 2, while the dataset and the experimental results are detailed in Section 3. The discussion of the results is given in Section 4, and the conclusion of the current research is outlined in Section 5.

\section{Proposed Methodology}

In this paper, a unique system that is based on selected deep features and Bayesian optimization based SVM classifier is proposed for classification of brain tumors. Extensive experimental studies were carried out for the determination of feature selection and classifier parameters. In addition, the wellknown CNN architectures were examined to identify the effects on the classification performance. Finally, a brain tumor dataset named Figshare, which is widely used in the literature, was used in experimental studies, and the accuracy scores were used for evaluation of the performance of the system proposed. The flowchart of the system proposed is presented in Fig. 1.

The proposed system incorporates four phases: preprocessing, feature extraction, feature selection and classification. These stages are detailed below under subheadings. 


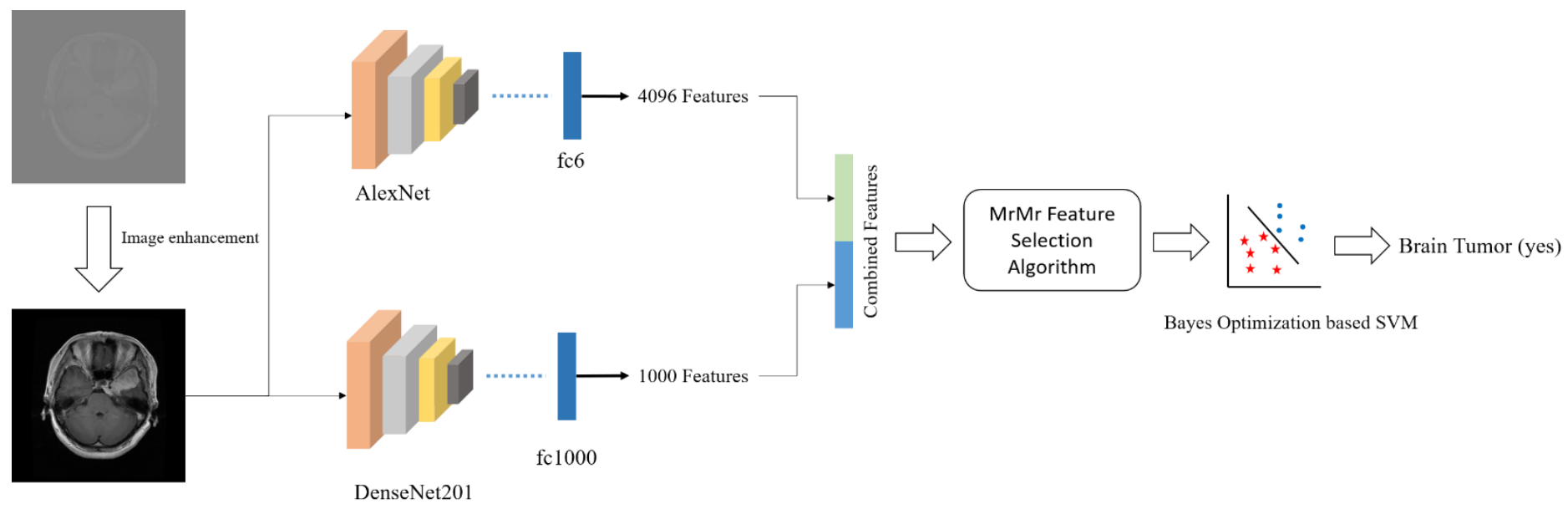

Figure 1 Flowchart of proposed model

\subsection{Pre-processing Phase}

In the pre-processing phase, brain MRI images are enhanced using max-min algorithm (Algorithm 1). Thus, an MRI image with a black background and a more prominent edge was obtained. Procedure of the proposed pre-processing phase is shown in Algorithm 1.

\section{Algorithm 1. The pseudo-code of the proposed pre-processing.}

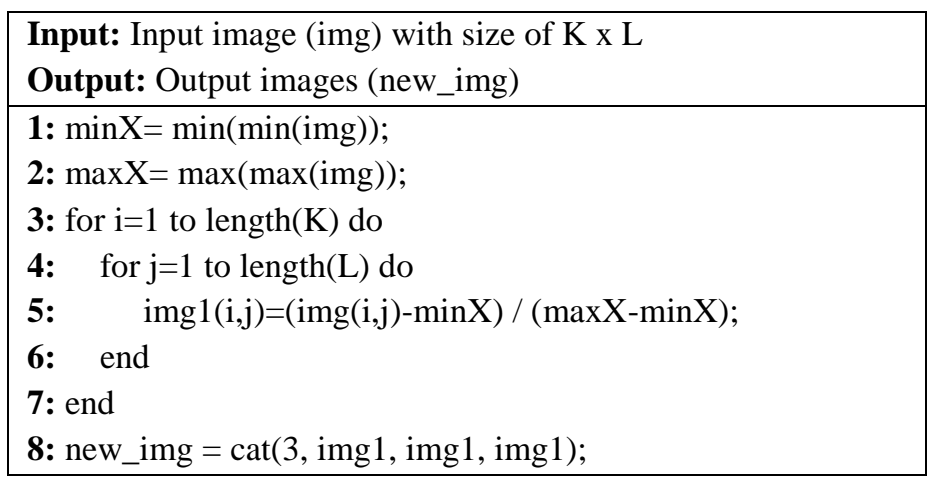

\subsection{Feature Extraction Phase}

In feature extraction phase, pre-trained architectures based on transfer learning were used for recognition of Brain MR images. The transfer learning approach is the adaptation of pretrained CNN-based deep architectures that use the learned weights to solve another problem. In this study, AlexNet, and DenseNet201 architectures, which are pre-trained CNN models with different characteristics, were used. The characteristics of these architectures are presented in Table 1.

Table 1. The characteristics of the deep architectures

\begin{tabular}{|l|c|c|c|}
\hline \multicolumn{1}{|c|}{ Name } & Depth & $\begin{array}{c}\text { Size } \\
\text { (MB) }\end{array}$ & $\begin{array}{c}\text { Parameters } \\
\text { (Millions) }\end{array}$ \\
\hline $\begin{array}{l}\text { AlexNet (Krizhevsky } \\
\text { et al., 2012) }\end{array}$ & 8 & 227 & 61 \\
\hline $\begin{array}{l}\text { DenseNet201 (Huang } \\
\text { et al., 2018) }\end{array}$ & 201 & 77 & 20 \\
\hline
\end{tabular}

These architectures shown in Table 1 were trained using the ImageNet dataset that had a very large collection of annotated images designed for developing machine learning methods. AlexNet architecture developed by Krizhevsky et al. consists of 25 layers: convolution, pooling layer, ReLU layer, and fully connected layer. The general structure of this architecture is shown in Fig. 2.

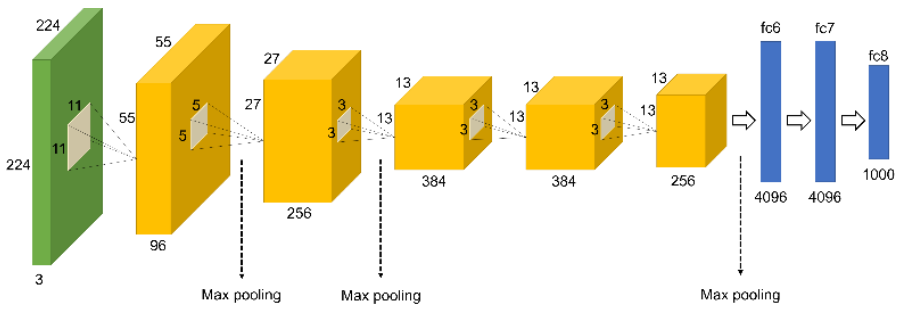

Figure 2 The structure of AlexNet architecture

The DenseNet network architecture is that the features obtained from the previous layers are directly combined with much more advanced layers (Fig. 3). Thus, the features obtained in the first layers are protected. The DenseNet201 network which one of the versions of this new approach has become a high-performance and multi-layer network model (Huang et al., 2018).

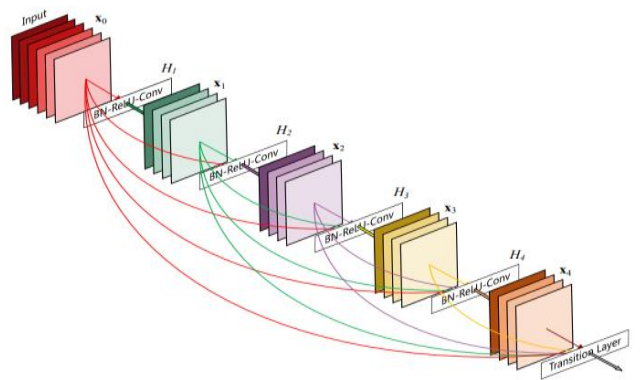

Figure 3 Inter-connection model of DenseNet (Huang et al., 2018) architecture

In this study, 4096, and 1000 deep features were extracted from fully connected layers of AlexNet, and DenseNet201 architectures, respectively. Then, deep features obtained from deep architectures with different structures were combined. Procedure of the proposed feature extraction phase is shown in Algorithm 2.

\subsection{Feature Selection Phase}

In feature selection phase, it is an important process to select the best feature subset from feature vectors for overcoming classification problems. The feature selection process has an important advantage of reducing the processing 
time by decreasing the number of features (Yaslan \& Cataltepe, 2009; Demir et al., 2020). In this study, it is aimed to determine better and distinctive features from combined deep features obtained during the feature extraction stage by using $\mathrm{MrMr}$ algorithm. This MrMr method is a filtering algorithm that tries to select the most relevant attributes with the class tags, while minimizing the redundancy between the selected attributes, simultaneously (Yaslan \& Cataltepe, 2009; Gulgezen et al. 2009; Toğaçar et al., 2020a).

\section{Algorithm 2. The pseudo-code of the proposed feature extraction.}

Input: Input image (img) with size of K x L x 3
Output: Deep features (feat)
1: img1=imresize (img, [224 224]);
2: img2=imresize (img, [227 227]);
3: Net_Parameters1= load_model (AlexNet, pretrained=true);
4: Net_Parameters2= load_model (DenseNet201,
pretrained=true);
5: Features1 = activations (Net_Parameters1, img2, 'fc6');
6: Features2 = activations (Net_Parameters2, img1, 'fc1000');
7: feat=[ Features1; Features2];

The MrMr based feature selection method used in this study are includes 2 basic steps. There: (1) The weights of the deep features are calculated, (2) sort weight scores, and select features as much as specific $t$ value. The selection procedure including these steps is demonstrated in Algorithm 3.

Algorithm 3. The pseudo-code of the proposed feature extraction.

Input: Deep features (dfeat), label (lab), and t value (selected
features)
Output: Selected features (feat)
1: w= fscmrmr (dfeat,lab);
2: for $i=1$ to t do
3: feat $(:, i)=d f e a t(:, w(i)) ;$
4: end for i

\subsection{Classification Phase}

In classification phase, SVM classifier was used for training of selected deep features. SVM method is a statistical algorithm used in classification problems (Cortes \& Vapnik, 1995). This method places the features on the coordinate plane and then performs the classification process by selecting the best hyperplane that can distinguish the two classes. Hyperplane parameters with two variables such as weight vector (w) and trend value (b) are adjusted to maximize symmetrical spacing between classes (Turkoglu \& Hanbay, 2019). The working principle of the SVM classifier is shown in Fig. 4.

Equation (1) aims to minimize the problems arising during the classification process. In Equation (2), $x_{i}$ ve $y_{i}$ represent features. This equation makes an estimate to determine the class of data (Cortes \& Vapnik, 1995; Turkoglu, 2020; Toğaçar et al., 2020b).

$$
\min \frac{1}{2}\|w\| \|^{2}
$$

$y_{i}\left(w^{T} \cdot x_{i}+b\right) \geq 1, i=1, \ldots, n$

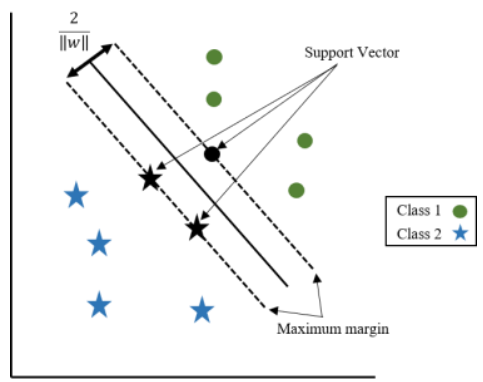

Figure 4 General structure of SVM classifier (Turkoglu, 2020)

In this study, the SVM classifier was preferred due to the fact that it is easy to apply, has high generalization performance, and is effective in high dimensional feature vectors. Additionally, the SVM method includes parameters such as Box constraint level, Kernel scale, and Kernel Function. The change in these parameter values significantly affects the classification performance. For this reason, it is aimed to select the best performance parameters of the SVM classifier by using Bayes optimization method (Pelikan et al., 1999), which is widely used and is faster than other optimization methods. A sample demonstration of these operations shown in Fig. 5.

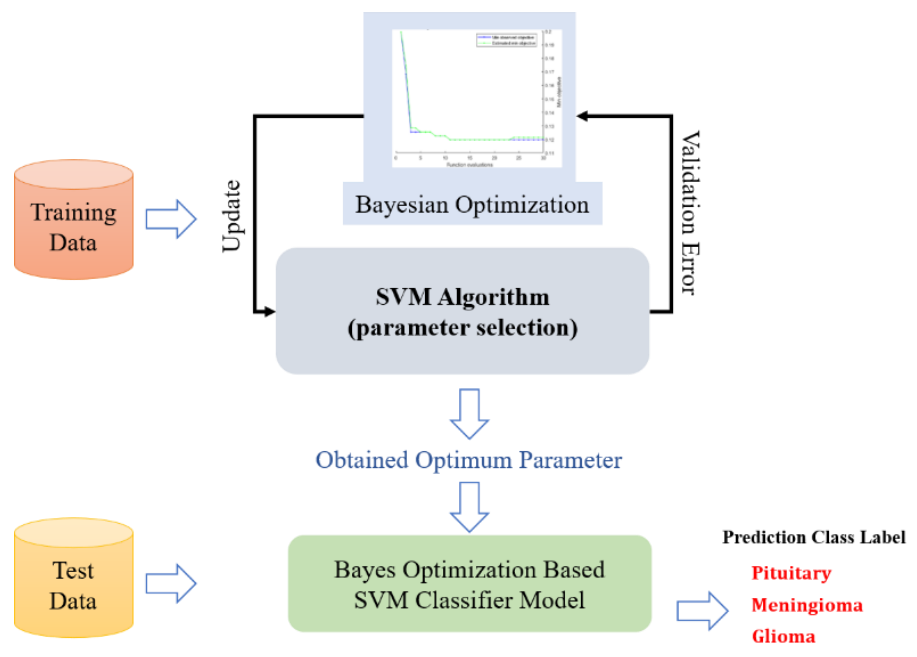

Figure 5 Bayes optimization based SVM classifier model

As can be understood from Fig. 5, the best parameters of the Bayes optimization based SVM classifier were selected for the training set and the performance of the system proposed for the test set was calculated using these parameters. In current study, the optimized hyperparameters and hyperparameters search ranges of SVM classifier based on Bayesian optimization are listed in Table 2. Additionally, the MATLAB Classification Learner toolbox is used for the application of Bayesian and SVM methods.

Table 2. The optimized hyperparameters and search ranges

\begin{tabular}{|l|l|l|l|}
\hline $\begin{array}{l}\text { Kernel } \\
\text { function }\end{array}$ & $\begin{array}{l}\text { Box } \\
\text { constraint } \\
\text { level }\end{array}$ & $\begin{array}{l}\text { Multiclass } \\
\text { method }\end{array}$ & $\begin{array}{l}\text { Standardize } \\
\text { data }\end{array}$ \\
\hline $\begin{array}{l}\text { Gaussian } \\
\text { Linear } \\
\text { Quadratic } \\
\text { Cubic }\end{array}$ & $\begin{array}{l}\text { [0.001- } \\
1000]\end{array}$ & $\begin{array}{l}\text { One-vs-One } \\
\text { One-vs-All }\end{array}$ & true / false \\
\hline
\end{tabular}




\section{Experimental Studies}

The Bayesian optimization based deep classifier proposed in this study was created using MATLAB software. In the experimental studies, a computer with a Nvidia M4000 GPU with $8 \mathrm{~GB}$ of memory was utilized. Additionally, using the random separation approach, $80 \%$ of the data was used for training and the rest for testing. This procedure was repeated only once, and the same training/test sets were used in all experimental studies. The code of the proposed model was shared at https://github.com/mturkoglu23/Brain-TumorDetection.

The experimental results and dataset are explained in the following subsections.

\subsection{Dataset}

In this paper, publicly available brain tumor dataset named Figshare (Cheng, 2018) was used. This dataset, developed by Cheng in 2017, contains 3064 brain MRI images. In addition, Figshare dataset includes three types of brain tumors namely pituitary, meningioma, and glioma. These brain tumor types are illustrated in Fig. 6.

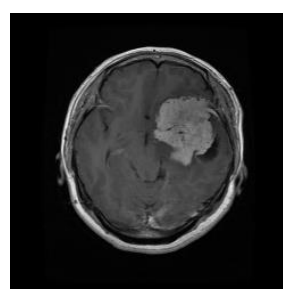

(a)

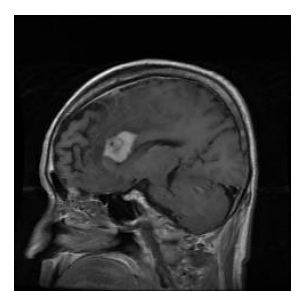

(b)

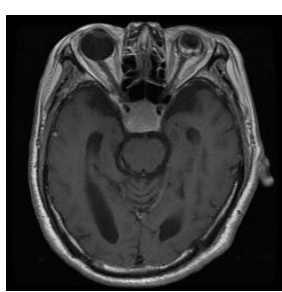

(c)
Figure 6 Types of Brain Tumors, a) Meningioma, b) Glioma, c) Pituitary.

\subsection{Results}

In this section, pretrained $\mathrm{CNN}$ models are adapted to the classification of brain tumors using a fine-tuning based on the transfer learning approach. The fine-tuning method is focused on the transfer of new layers to our classification mission, instead of the last three layers of the pre-trained networks. These layers are a fully connected, a softmax, and a classification. In this study, accuracy scores were calculated using this approach for AlexNet, and DenseNet201 architectures. The network parameters used for these deep architectures are given in Table 3. Accuracy scores achieved from this experimental works are shown in Table 4.

Table 3. The deep network parameters used in current study.

\begin{tabular}{|r|l|l|}
\hline Mini-batch size & $:$ & 8 \\
\hline Maximum epoch number & $:$ & 10 \\
\hline Weight decay factor & $:$ & 0.01 \\
\hline Initial learning rate & $:$ & 0.001 \\
\hline Optimization method & $:$ & $\begin{array}{l}\text { SGDM (Stochastic Gradient } \\
\text { Descent with Momentum) }\end{array}$ \\
\hline
\end{tabular}

Table 4. Accuracy scores (\%) of fine-tuned deep architectures

\begin{tabular}{cc}
\hline AlexNet & DenseNet201 \\
92.17 & 89.72 \\
\hline
\end{tabular}

As is apparent from Table 4, the highest accuracy score between deep architectures based on fine-tuning process was 92.17\% with the AlexNet architecture. In addition, the accuracy score of DenseNet201 architecture was obtained as $89.72 \%$. As another experimental work, features were extracted from fully connected layers of these deep architectures, and then deep features were fed into an SVM classifier. The confusion matrixes (accuracy scores) achieved from this experimental works are given in Figure 7.

(a)

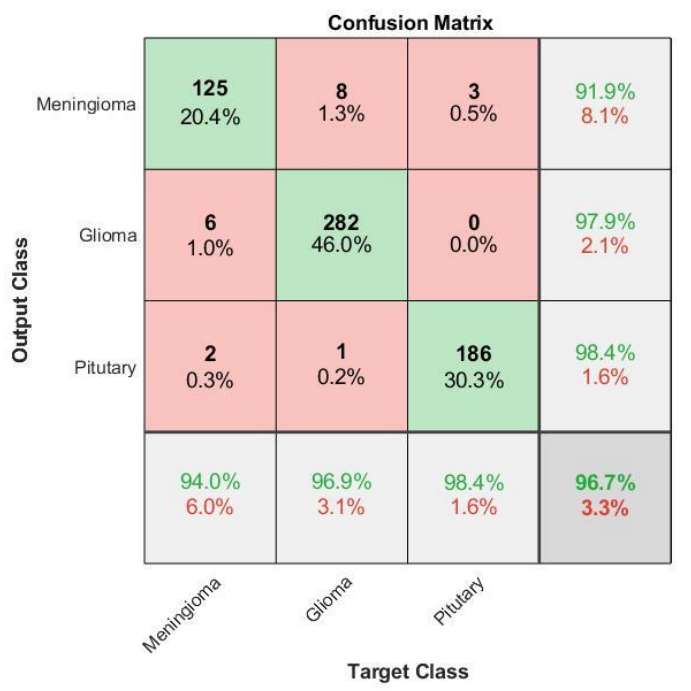

(b)

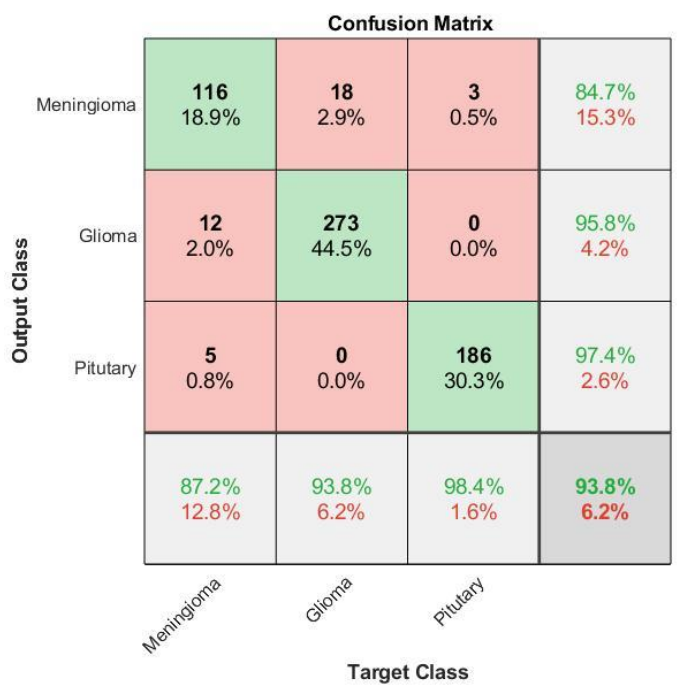

Figure 7 Confusion matrix of deep models, a) AlexNet, b) DenseNet201

As can be seen in Figure 7, the highest accuracy score between deep architectures based on deep feature extraction approach was $96.74 \%$ with the AlexNet architecture. In addition, the accuracy score of DenseNet201 architecture was obtained as 93.8\%. According to the results given in Table 4 and Figure 7, performance results based on deep features and SVM classifier for all deep architectures is more successful than fine-tuning. For these reasons, deep feature extraction and SVM approaches used in the remainder of the study.

In the second experiment, combinations of AlexNet, and DenseNet201 deep architectures were evaluated. In accordance with this purpose, features obtained from deep architectures were combined, and their performances were calculated using an SVM classifier. The analysis results of the combined deep features are given in Figure 8. According to these results, the 
highest accuracy score among combined deep networks was achieved as $97.39 \%$ using a combination of AlexNet, and DenseNet201 architectures. In addition, the accuracy score of combined deep networks was a $1 \%$ performance increase from the AlexNet architecture which has the highest performing. As a result, the combined method used in the second experiment improved the accuracy score of the individual deep architecture. The confusion matrixes for the combined deep networks are shown in Fig. 8.

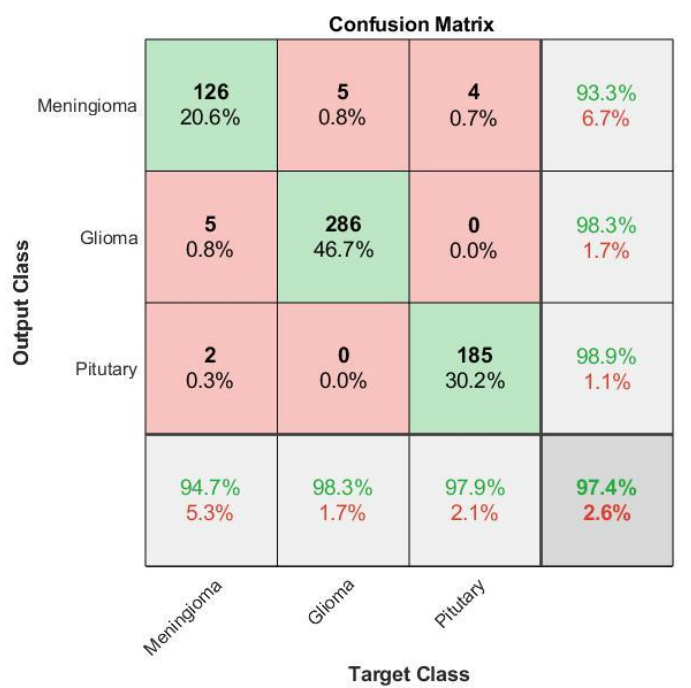

Figure 8: Confusion matrix of combined deep models

In the last experimental study, a combination of selected deep features and Bayesian optimization based SVM classifier is proposed for the classification of brain tumors. In line with this purpose, features were obtained from deep architectures with different structures, and then these deep features were combined. More meaningful and effective features were selected from the combined features obtained using $\mathrm{MrMr}$ method and trained with Bayes optimization based SVM model. Consequently, the performance of the features selected in different numbers were calculated, and these results are presented in Fig. 9.

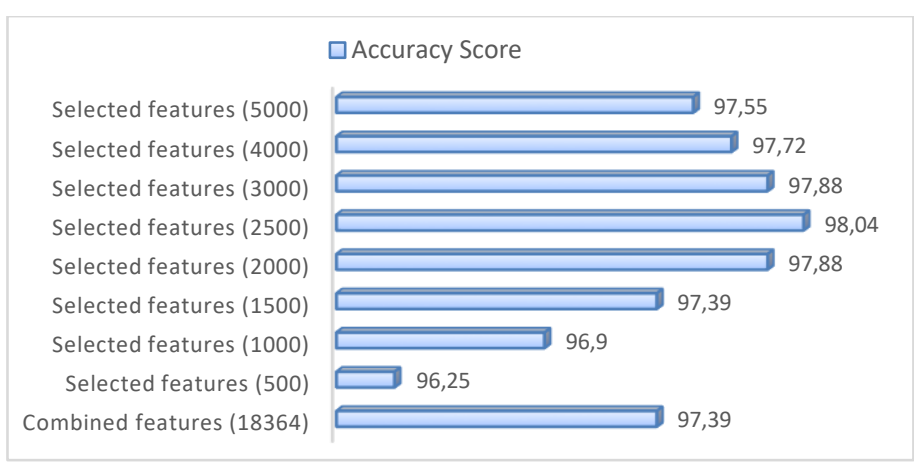

Figure 9 The accuracy scores of selected combined deep features

As can be understood from Fig. 9, the distinguishing features (except for 500 and 1000 selected features) selected using MrMr algorithm are observed to be more successful than the raw features. Moreover, the 500 features obtained from the combined deep properties have the worst accuracy at $96.25 \%$. The best accuracy score of the model proposed based on $\mathrm{MrMr}$ algorithm for classifying brain tumors was achieved as $98.04 \%$ as selected 2,500 effective features. Additionally, 2000, and 3000 most relevant features selected from deep features have approximately the same accuracy. The confusion matrix of the best performance $(98.04 \%)$ for the model proposed are shown in Fig. 10.

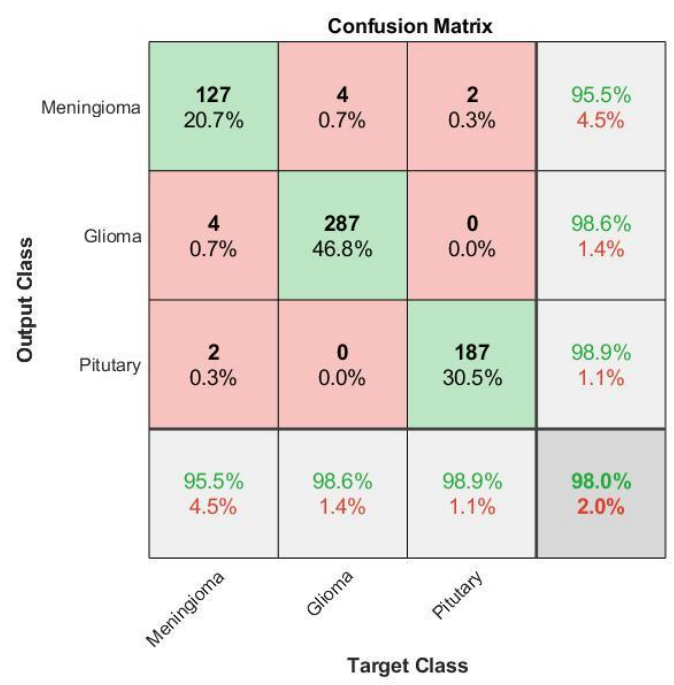

Figure 10 Confusion matrix and ROC curve of best accuracy score obtained from proposed model

In the proposed model, the highest performance $(98.04 \%$ 2500 deep features) using the Bayesian optimization method for SVM classifier was achieved with kernel function (Cubic), box constraint level (244.4749), and multiclass method (one-vs-all). The minimum classification error plot of the Bayesian optimization process based SVM classifiers is presented in Fig. 11 .

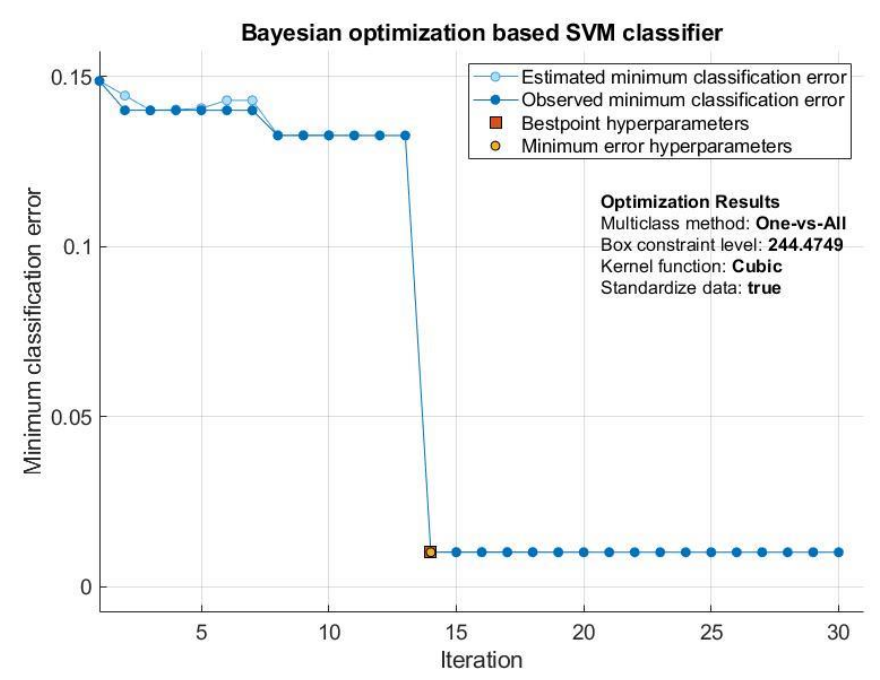

Figure 11 Bayesian optimization based SVM classifier-minimum classification error plot

As can be seen in Figure 11, 30 iterations were executed for the Bayesian optimization process based on the SVM classifier. The best-point of hyperparameters is accomplished at the end of the 14th iteration.

\section{Discussion}

In this paper, the Bayesian optimization based SVM classifier proposed, and selected $\mathrm{CNN}$ based deep features were compared to the existing systems in the literature, and the results are presented in Table 8. 
Table 8. Comparison of the existing studies with model proposed

\begin{tabular}{|c|c|c|c|}
\hline References & Feature extraction & Classification & Accuracy \\
\hline Ari et al. (2020) & AlexNet and VGG16 & ELM & $97.64 \%$ \\
\hline Cheng et al. (2016) & Local features using Fisher Vector & SVM & $94.68 \%$ \\
\hline Abir et al. (2018) & GLCM & PNN & $83.33 \%$ \\
\hline Afshar et al. (2019) & Capsule networks (CapsNet) & & $86.56 \%$ \\
\hline Cheng et al. (2015) & Bag of words & SVM & $91.28 \%$ \\
\hline Deepak and Ameer (2019) & GoogleNet & SVM & $97.1 \%$ \\
\hline Kaur and Gandhi (2020) & Fine-tuned AlexNet & & $96.95 \%$ \\
\hline Ayadi et al. (2020) & DSURF and $\mathrm{HoG}$ & SVM & $90.27 \%$ \\
\hline Pashaei et al. (2018) & $\mathrm{CNN}$ & ELM & $93.68 \%$ \\
\hline Swati et al. (2019) & Fine-tune VGG19 & & $94.80 \%$ \\
\hline Deepak and Ameer (2020) & $\mathrm{CNN}$ & SVM & $95.82 \%$ \\
\hline Bodapati et al. (2020) & Xception and InceptionResNetV2 & Softmax & $95.23 \%$ \\
\hline Proposed model & AlexNet and DenseNet201 & Bayes optimization based SVM & $98.04 \%$ \\
\hline
\end{tabular}

Table 8 summarizes the previous studies and gives accuracy scores. Among the previous studies using traditional machine learning methods. (Cheng et al., 2015; Cheng et al., 2016; Abir et al., 2018; Ayadi et al., 2020) studies for classification of brain tumors applied feature extraction methods such as Fisher Vector, GLCM, Bag of words, and DSURF/HoG, respectively. In addition, all these studies used the SVM classifier. The highest accuracy score among these works based on traditional methods is achieved as $94.68 \%$ by Cheng et al. (2016). On the other hand, the previous works based on pre-trained deep architectures used two approaches which are deep feature extraction and transfer learning (fine-tuned). (Deepak \& Ameer, 2019; Ari et al., 2020; Bodapati et al., 2020) studies which used deep feature extraction approach presented combination of pre-trained deep models and SVM/ELM/Softmax classifiers. The highest accuracy score among these studies is obtained as $97.64 \%$ by Ari et al. (2020). (Swati et al., 2019; Kaur \& Gandhi, 2020) works based on transfer learning (fine-tuned) used AlexNet, and VGG19, respectively. The highest accuracy score among these studies is obtained as $96.95 \%$ by Kaur and Gandhi (2020). According to all these results, deep learning-based studies for classification of brain tumors demonstrated higher performances than those of traditional machine learning based studies, as depicted in Table 8. Moreover, as it can be understood from Table 8, the accuracy score of the model proposed in the current study are observed to be more successful than those of the existing studies. follows:

The advantages and limitations of the model proposed are as Advantages:

- The proposed model selected features which have high performance using feature selection algorithm.

- The best classifier parameters of SVM method were selected by using Bayes optimization algorithm.

- The combined and selected deep features ensemble model achieved a higher accuracy score than that of pre-trained deep architectures.

Limitations:

- It can be seen as a limitation of the study to find optimal selected features using the MrMr algorithm.

\section{Conclusion}

Proposed in this study is a hybrid model based on the Bayesian optimization based SVM classifier, four pre-trained CNN models and $\mathrm{MrMr}$ feature selection method for classification of brain tumors. In line with this purpose, a publicly available brain tumor dataset consisting of 3024 MRI images in total was used. The method proposed entailed three experiments. In the first experiment, pre-trained deep architectures based on the transfer learning approach were used as feature extractors, and representative features were obtained from brain MRI images. In the second experiment, MrMr feature selection method was used to select distinctive and more effective features from combined deep features. Finally, Bayesian optimization method was used for SVM classifier parameters and hyperparameters with the best performance were selected. According to the experimental results, the best accuracy score of $98.04 \%$ was achieved using model proposed for classification of brain tumors.

In future works, a real-time web-based system is planned to be developed, which will aim to support health professionals to detect brain tumors and other diseases. In addition, other $\mathrm{CNN}$ based models, machine learning classifier and attention modules will be investigated to help classification of brain tumors.

\section{References}

Abir, T.A., Siraji, J.A., Ahmed, E., \& Khulna, B. (2018). Analysis of a novel MRI based brain tumour classification using probabilistic neural network (PNN). Int. J. Sci. Res. Sci. Eng. Technol., 4(8), 65-79.

Afshar, P., Mohammadi, A., \& Plataniotis, K.N. (2018). Brain tumor type classification via capsule networks. arXiv preprint: arXiv:1802.10200.

Afshar, P., Plataniotis, K.N., \& Mohammadi, A. (2019). Capsule networks for brain tumor classification based on MRI images and coarse tumor boundaries. In ICASSP 20192019 IEEE International Conference on Acoustics, Speech and Signal Processing (ICASSP), pp. 1368-1372.

Amin, J., Sharif, M., Raza, M., Saba, T., \& Rehman, A. (2019). Brain Tumor Classification: Feature Fusion. In 2019 
International Conference on Computer and Information Sciences (ICCIS), pp. 1-6.

Amin J., Sharif, M., Gul, N., Yasmin, M., \& Shad, S.A. (2020). Brain tumor classification based on DWT fusion of MRI sequences using convolutional neural network. Pattern Recognition Letters, 129, 115-122.

Ari, A. (2019). Detection and classification of brain tumors from MR images based on deep learning. PhD. Thesis, Inonu University, Malatya, Turkey.

Ari, A., Alcin, O.F., \& Hanbay, D. (2020). Brain MR Image Classification Based on Deep Features by Using Extreme Learning Machines. Biomedical Journal of Scientific \& Technical Research, 25(3), 1937-1944.

Ayadi, W., Charfi, I., Elhamzi, W., \& Atri, M. (2020). Brain tumor classification based on hybrid approach. The Visual Computer, 1-11.

Bodapati, J.D., Shaik, N.S., Naralasetti, V., \& Mundukur, N.B. (2020). Joint training of two-channel deep neural network for brain tumor classification. Signal, Image and Video Processing, 1-8.

Cheng, J., et al. (2015). Enhanced performance of brain tumor classification via tumor region augmentation and partition. PloS one, 10(10), e0140381.

Cheng, J., et al. (2016). Retrieval of brain tumors by adaptive spatial pooling and fisher vector representation. PLoS ONE, 11(6), e0157112.

Cheng, J. (2018). Brain tumor dataset (Figshare dataset), https://doi.org/10.6084/m9.figshare.1512427.v5. Accessed 30 May 2018.

Cortes, C., \& Vapnik, V. (1995). Support-vector networks, Machine Learning, 20(3), 273.

Deepak, S., \& Ameer, P.M. (2019). Brain tumor classification using deep CNN features via transfer learning. Computers in biology and medicine, 111, 103345 .

Deepak, S., \& Ameer, P.M. (2020). Automated Categorization of Brain Tumor from MRI Using CNN features and SVM. Journal of Ambient Intelligence and Humanized Computing, 1-13.

Demir, F., Turkoglu, M., Aslan, M., \& Sengur, A. (2020). A new pyramidal concatenated $\mathrm{CNN}$ approach for environmental sound classification. Applied Acoustics, 170, 107520.

Gulgezen, G., Cataltepe, Z., \& Yu, L. (2009). Stable feature selection using MRMR algorithm. In 2009 IEEE 17th Signal Processing and Communications Applications Conference, pp. 596-599.

Huang, G., Liu, Z., Van Der Maaten, L., \& Weinberger, K.Q. (2018). Densely connected convolutional networks. In Proceedings of the IEEE conference on computer vision and pattern recognition, pp. 4700-4708.

Kaur, T., \& Gandhi, T.K. (2020). Deep convolutional neural networks with transfer learning for automated brain image classification. Machine Vision and Applications, 31, 1-16.

Krizhevsky, A., Sutskever, I., \& Hinton, G.E. (2012). Imagenet classification with deep convolutional neural networks. InAdvances in neural information processing systems, 1097-1105.

Pashaei, A., Sajedi, H., \& Jazayeri, N. (2019). Brain tumor classification via convolutional neural network and extreme learning machines. In 2018 8th International conference on computer and knowledge engineering (ICCKE), pp. 314319.

Pelikan, M., Goldberg, D., \& Cantú-Paz, E. (1999). BOA: The Bayesian optimization algorithm. In Proceedings of the genetic and evolutionary computation conference GECCO99, 1, 525-532.

Rehman, A., Naz, S., Razzak, M.I., Akram, F., \& Imran, M. (2020). A deep learning-based framework for automatic brain tumors classification using transfer learning. Circuits, Systems, and Signal Processing, 39(2), 757-775.

Swati, Z.N.K. et al. (2019). Brain tumor classification for MR images using transfer learning and finetuning. Computerized Medical Imaging and Graphics, 75, 34-46.

Toğaçar, M., Ergen, B., \& Cömert, Z. (2020a) Detection of lung cancer on chest CT images using minimum redundancy maximum relevance feature selection method with convolutional neural networks. Biocybernetics and Biomedical Engineering, 40(1), 23-39.

Toğaçar, M., Ergen, B., \& Cömert, Z. (2020b). Waste classification using AutoEncoder network with integrated feature selection method in convolutional neural network models. Measurement, 153, 107459.

Turkoglu, M., \& Hanbay, D. (2019). Plant disease and pest detection using deep learning-based features. Turkish Journal of Electrical Engineering \& Computer Sciences, 27(3), 1636-1651.

Turkoglu, M. (2020). COVIDetectioNet: COVID-19 diagnosis system based on X-ray images using features selected from pre-learned deep features ensemble. Applied Intelligence, $1-14$.

Yaslan, Y., \& Cataltepe, Z. (2009). Audio genre classification with co-mrmr. In 2009 IEEE 17th Signal Processing and Communications Applications Conference, pp. 408-411. 